\title{
Reseña: La empresa en el siglo XXI. Un estudio multidisciplinar de carácter jurídico empresarial. Eduardo Carlos Dittmar (2019)
}

Dra. Patricia Vargas Portillo

ORCID: 0000-0002-0226-3053

ESIC Business \& Marketing School, España

Correo: jennypatricia.vargas@esic.edu

Eduardo Carlos Dittmar es vicedecano de investigación de EAE Business School, así como editor de la revista Harvard Deusto Business Research. Su investigación presenta carácter interdisciplinar, pudiendo calificarse de frontera entre las ciencias empresariales y el mundo jurídico. Una formación jurídica que supere la perspectiva meramente normativa e incluya otras dimensiones —como, entre otras, la económica, empresarial, política, histórica, sociológica o filosófica- suministraría al operador jurídico diferentes instrumentos que le darían la oportunidad de comprender, con una mayor amplitud, los fenómenos sociales y aplicar las normas respecto a la realidad social.

La monografía coordinada por Dittmar (2019) incluye un sugerente elenco de capítulos que abordan temas muy heterogéneos entre sí. Todos ellos tienen en común varias notas características. Entre ellas, podemos destacar, por un lado, el hecho de ser estudios de carácter transversal y, por otro, analizar temas de vanguardia. Como decían Balkin y Levinson ${ }^{1}$, a fecha de hoy no es posible vislumbrar la teoría jurídica sin considerar la impronta de la interdisciplinariedad.

El prólogo, a cargo de Javier Pijoan, director general en Bacardi Limited Iberia, presenta de manera somera la obra, dando cuenta de los avances que representa. Como el prologuista determina, se examinan los acontecimientos desde una perspectiva holística,

\footnotetext{
1 Balkin, J. M., y Levinson, S. (2006). Law and the Humanities: An Uneasy Relationship. Yale Journal of Law \& the Humanities, 18, 155-187.
} 
actual, original y de una notable aplicación práctica. Presenta una lectura sencilla y fluida, pero su contenido es riguroso. Los asuntos analizados son de temática amplia e inclusiva, refiriéndose a estrategias y transformaciones suficientemente innovadoras.

El primero de los capítulos se refiere al estudio del marco conceptual de la empresa multinacional. En sus comienzos alude a los caracteres y efectos que presenta la globalización. Resultan muy sugerentes las apreciaciones, tanto positivas como negativas, que son predicables respecto a tal fenómeno. De hecho, la reciente pandemia sufrida a nivel global ha dado lugar a que nos planteemos si la globalización ha de mantenerse o desaparecer. La COVID-19 representa una amenaza más de las que afectan a la humanidad. La diferencia, más visible en la actualidad reside en que vivimos en un mundo muy interconectado y vertiginoso al que denominamos globalización, por lo que se alzan voces muy contrarias a esta. Desde la óptica del Derecho comercial, se refiere a interesantes teorías de aplicación a esta cuestión. La empresa internacionalizada ostenta prerrogativas a considerar frente a las no internacionalizadas.

El siguiente capítulo se refiere a la innovación, a escala mundial, y su repercusión en España. El presente exhibe una óptica jurídica de notable interés que se apoya sobre un plano marcadamente empírico. Una afirmación que puede efectuarse es que la innovación española se ve ciertamente afectada en tiempos de crisis. Si bien en España se innova, se hace por debajo de la media del entorno. Si tuviéramos que mencionar a los referentes en esta materia, tendríamos que referirnos a Suecia, Dinamarca, Alemania y Finlandia. Las patentes y los modelos de utilidad constituyen manifestaciones obvias de la innovación. Las ideas innovadoras son el pilar sobre el que se sustentan un gran número de empresas florecientes. Pero las ideas per se presentan un limitado valor económico. Es necesario desarrollarlas, convertirlas en productos o servicios transformadores y comercializarlas para poder lograr los beneficios inherentes a la innovación. Todo ello, como no podía ser de otra manera, con la preceptiva protección jurídica que da la Propiedad Intelectual e Industrial.

El capítulo tercero se refiere a un tema de extraordinaria actualidad y proyección de futuro: el Derecho digital, el cambio de paradigma y la transformación tecnológica. Asimismo, se presta una atención de primer orden a las smart cities que, como la realidad pone de manifiesto, es un fenómeno que ha venido para quedarse. Cada vez un mayor número de ciudadanos se traslada del mundo rural al urbano. En este sentido, según la ONU, en 2050 el $70 \%$ de la población vivirá en la ciudad. Ahora bien, no solo debería 
avanzarse en tecnología y sostenibilidad, dado que también debe atenderse a la regulación. Así, a título de ejemplo, hay tecnologías de reconocimiento facial que pueden regular la temperatura de una casa cuando se accede a ella, aunque es posible que la ordenación legal — por motivos de protección de datos_ - impida implantarlas.

Este capítulo también versa sobre otros aspectos, no menos significativos, como el big data; el comercio electrónico; la propiedad intelectual $\mathrm{y}$, finalmente, las criptomonedas. Todo ello ha de vincularse con la amenaza de las denominadas Fintech y las Big tech. Dada la relevancia que está protagonizando, hubiera sido deseable un mayor análisis de la incidencia que el blockchain o la cadena de bloques está desempeñando en numerosos ámbitos de la vida social. El primer aspecto que ha de considerar una organización es qué modalidad de cadena de bloques es más adecuada: privada o pública. Habida cuenta de los retos que implican la cadena de bloques de carácter público, las organizaciones tienen una mayor tendencia a focalizarse en redes privadas, que dan una mayor oportunidad de ostentar más control sobre quién puede adherirse a la red.

La regulación de la inteligencia artificial se analiza en el capítulo cuarto. El contenido es apasionante en toda su extensión. En primer lugar, se aborda la afección de determinados derechos fundamentales. A continuación, se refiere a las escuchas automatizadas que hoy día acontecen en los smartphones - a través del micrófono que posteriormente da origen a una publicidad comportamental- y en dispositivos inteligentes como Alexa o Google Home, que también se basan en los perfiles de los usuarios. Especial atención merece el marco legal de la captación del audio, cuyas prerrogativas para la industria son múltiples. Igual consideración merecen las apreciaciones relativas a la jurisprudencia constitucional y la legislación, nacional y comunitaria, que se ha aprobado en materia de protección de datos. Ahora bien, la legislación debe entenderse completada por la ética que entra en juego a través de los denominados códigos de conducta. Estos últimos representan una manifestación de la autodisciplina de la industria.

Vinculado parcialmente con la última cuestión apuntada, se enmarca el capítulo quinto. Este se ocupa del examen de los códigos de conducta en el ámbito digital. Inicialmente se refiere al examen, en un sentido amplio, del fenómeno de la autorregulación. A continuación, alude a la incidencia de la Ley 3/1991 de competencia desleal. Todo cuanto se plantea se enmarca en las resoluciones realizadas por organismos de autodisciplina respecto a terceros no adheridos al sistema. En tal supuesto pueden 
suscitarse daños muy significativos desde el punto de vista de la reputación del prestador de servicios no adherido a estos sistemas de autorregulación. Quizás, habría sido pertinente que el autor se hubiera referido, con carácter complementario, a las cuestiones que se plantean en materia de defensa de la competencia.

El siguiente capítulo alude al ámbito logístico y los vínculos que tal cuestión ostenta respecto a la cadena de bloques y los contratos inteligentes. El mencionado blockchain está progresivamente más presente en el transporte y la logística. Esta novedosa herramienta tecnológica va a revolucionar el devenir del comercio internacional, aunque también del transporte de mercancías. Cuando se dispone de un sistema fundamentado en la cadena de bloques se puede dejar constancia, a tiempo real, del estado de un producto con independencia de la etapa en la que la cadena logística esté y quién lo esté operando.

El capítulo séptimo se refiere a las criptomonedas, en el que se analiza si es el futuro del dinero o el final de una burbuja. Existe una enorme multiplicidad de monedas inteligentes, pero todas ellas tienen en común el hecho de que están sometidas a una extrema volatilidad. Aunque la más conocida es Bitcoin, existen una multitud que crece a diario (además de esta podemos citar otras como Litecoin, Ethereum, Monero, Bitcoin Cash y Dash). Bitcoin es muy segura, ya que no se han logrado crear bitcoins falsos, mientras que constantemente vemos intentos de falsificar dinero. Otra prerrogativa es que su creador consideró que en ningún caso se podrán superar los 21 millones de Bitcoin, a diferencia de las divisas usuales cuyos Estados pueden decidir poner en circulación más dinero. Cada vez un mayor número de transacciones comerciales se efectúan con monedas inteligentes, siendo su nivel de seguridad muy elevado. Hubiera sido oportuno un mayor detalle de la regulación legal que impera a nivel nacional, europeo e internacional tanto de las criptomonedas como de la cadena de bloques. Por lo que se refiere a España, el 10 de enero de 2020 finalizó el plazo de transposición de la Directiva comunitaria 2018/843 relativa al Blanqueo de Capitales que todavía no ha sido incorporada al Derecho español.

La alimentación saludable y la normativa legal que repercute en el consumo se desarrolla en el capítulo siguiente. A lo largo de su contenido se aborda qué significa la alimentación saludable y se examina la regulación legal que impera en el etiquetado de alimentos deteniéndose, en este sentido, en las alegaciones de salud y las tablas e información nutricional. Con buen criterio, se analizan algunas iniciativas del Ministerio de Sanidad y Consumo de España como la Estrategia para la nutrición, actividad física 
y prevención de la obesidad. Ahora bien, la parte de heterorregulación, establecida por parte del legislador, debiera haberse complementado con las iniciativas aprobadas por la industria como el Código PAOS de Autocontrol de la Publicidad.

El capítulo siguiente, vinculado con los Recursos Humanos y el Derecho laboral, se centra en la metodología que, en esta materia, ha sido implementada por Orange España. Una de las cuestiones que se abordaron, respecto a la adaptación del sistema Agile, fue la aplicación del Real Decreto Ley 8/2019, pues puede representar una dificultad sobre el plan de trabajo flexible y las políticas de conciliación que tienen implementadas, dirigidas a medir las personas por el esfuerzo que realizan y el resultado que obtienen. Para Orange, este extremo no ha implicado hándicap alguno pues, con sus políticas actuales de conciliación, resulta posible actuar conforme a la normativa legal. El teletrabajo, hasta hace muy poco, presentaba una utilización residual por parte de las empresas de un gran número de países.

Por lo que se refiere al ámbito europeo, los Estados que más recurrían a este eran los nórdicos, a diferencia de otros como España. Ahora bien, desde hace relativamente poco tiempo, se han sentado las bases para legislar el teletrabajo en base a que el fenómeno presenta una importante proyección de futuro. El desafío que tendría esta nueva normativa se puede resumir en dos aspectos. En primer lugar, instituir medidas para que la equiparación de derechos sea efectiva. En segundo lugar, fomentar que los agentes sociales convengan las medidas precisas para que la norma no constituya un obstáculo en el impulso del trabajo a distancia y del teletrabajo.

El último de los capítulos se refiere a Amazon y los importantes problemas de competencia desleal que esta gran empresa tecnológica suscita. Antes de hacer unas breves pinceladas al respecto, conviene detenerse en el concepto de competencia. Este último alude a la situación que concurre entre dos o más empresarios que actúan en el seno de un mismo sector del mercado y que ofertan bienes y/o servicios que buscan de satisfacer una misma necesidad del consumidor y/o usuario, pudiendo encontrar la situación del conflicto de intereses.

La Ley de Competencia Desleal salvaguarda la igualdad de los competidores, debiendo proceder en igualdad de condiciones sin poder hacer uso de enfoques concurrenciales aventajados que se hayan logrado gracias a la vulneración de las normas que disciplinan el mercado en cuestión. Para poder poner de manifiesto que está desarrollando prácticas abusivas debe estar fundamentado en evidencias sólidas. Pueden 
imperar elementos de juicio suficiente para considerar que Amazon presenta un comportamiento desleal contrario al buen funcionamiento del mercado que puede dejar en una situación de inferioridad a sus competidores al estar limitando la competencia. Su ventaja competitiva se apoya, entre otros aspectos, en la experiencia del usuario, la innovación en logística y la satisfacción y fidelización del cliente.

En suma, nos encontramos ante una obra de ineludible referencia por la interdisciplinariedad y heterogeneidad de contenidos de vanguardia con incidencia en el ámbito jurídico y empresarial.

\section{Ficha técnica}

Título: La empresa en el siglo XXI: Un estudio multidisciplinar de carácter jurídico empresarial

Coordinador: Eduardo Carlos Dittmar

Editorial: Aranzadi

Ciudad y país: Navarra, España

ISBN: 978-84-1308-916-4

Páginas: 299 